\title{
Balancing for Unstable Nonlinear Systems
}

\author{
J.M.A. Scherpen* \\ Department of Applied Mathematics, University of Twente \\ P.O. Box 217, 7500 AE Enschede, The Netherlands \\ e-mail: scherpen@math.utwente.nl
}

\begin{abstract}
A previously obtained method of balancing for stable nonlinear systems is extended to unstable nonlinear systems. The similarity invariants obtained by the concept of LQG balancing for an unstable linear system can also be obtained by considering a past and future energy function of the system. By considering a past and future energy function for an unstable nonlinear system, the concept of these similarity invariants for linear systems is extended to nonlinear systems. Furthermore the relation of this balancing method with the previously obtained method of balancing the coprime factorization of an unstable nonlinear system is considered. Both methods are introduced with the aim of using it as a tool for model reduction.
\end{abstract}

Keywords: balancing, nonlinear systems, HamiltonJacobi-Bellman equations, model reduction.

\section{Introduction}

Balancing is a well known subject in system theory. It started with a paper of Moore, [9], in 1981, where balancing for stable linear systems is introduced with the aim of using it as a tool for model reduction. The input and output energy of a system play an important role in this set up. Balancing for unstable linear systems has been treated by [6]. In this paper LQG balancing is introduced. LQG balancing can be used as a tool for model reduction of unstable linear systems and their LQG compensators. Furthermore, balancing of the coprime factorization of an unstable linear system is introduced in [8] and [11], where in [11] the relation with LQG balancing is given. Balancing for linear systems and the relation between LQG balancing and balancing of the coprime factorization is treated from another point of view by Weiland in [18].

Recently, balancing for stable nonlinear systems has been introduced in [14]. It is an extension of the idea of balancing for linear systems in the sense that the input and output energy of the nonlinear system are important to decide whether or not a state component is important for the model and may be deleted to obtain the reduced-order system. An extension of this method to unstable nonlinear systems by considering the normalized right coprime factorization of a nonlinear system can be found in [15]. This matches with the concept of balancing the coprime factorization of an unstable linear system as mentioned before.

In this paper we also consider unstable nonlinear systems, but now we propose a method to balance these systems based on a certain future and past energy function of the system, replacing the input and output energy functions which are important for balancing of a stable system. In the case of an unstable linear

\footnotetext{
'Supported by the Dutch Systems and Control Theory Network
}

system this method is the same as LQG balancing, which can be found in [6], [12] and [18]. Furthermore, we consider the relation with balancing of the normalized coprime factorization from [15].

In section 2 we give a brief review on LQG balancing and coprime balancing for linear systems. Section 3 contains a review on balancing for stable nonlinear systems and balancing of the coprime factorization of nonlinear systems. In section 4 we define HJB (Hamilton-Jacobi-Bellman) balancing and the HJB singular value functions. We propose a procedure to bring a nonlinear system into HJB balanced form, which is related to the procedure to balance stable nonlinear systems. Furthermore we study the relation of HJB balancing with balancing of the coprime factorization. Finally in section 5 we give some conclusions.

Throughout this paper we will use a fairly standard notation. We denote by $x^{T} x$ or $\|x\|^{2}$ the squared norm of a vector $x \in \mathbf{R}^{n}$. We say that $u:(-\infty, 0) \rightarrow \mathbf{R}^{m}$ is in $L_{2}(-\infty, 0)$ if $\int_{-\infty}^{0}\|u(t)\|^{2}$ $d t<\infty$. By $\frac{\partial L}{\partial x}(x)$ we denote the row-vector of partial derivatives of a differentiable function $L: \mathbf{R}^{n} \rightarrow \mathbf{R}$. Furthermore we denote by $x\left(t_{2}\right)=\varphi\left(t_{2}, t_{1}, x_{1}, u\right)$ the solution on time $t_{2}$ of the system $\dot{x}=f(x)+g(x) u$ with initial condition $x\left(t_{1}\right)=x_{1}$ and input $u:\left[t_{1}, t_{2}\right] \rightarrow \mathbf{R}^{m}$.

\section{Review for linear systems}

\subsection{LQG-balancing}

LQG-balancing for linear systems has been introduced in [6], and in [12] this concept is developed further. The set of invariants defined in these two papers are treated from another point of view in [18]. First we will give a review of the formulation of [6] and [12]. LQG compensation is formulated for a minimal statespace system

$$
\dot{x}=A x+B u+B d, \quad y=C x+v
$$

where $u \in \mathbf{R}^{m}, x \in \mathbf{R}^{n}, y \in \mathbf{R}^{p}$ and $d$ and $v$ are Gaussian white noise processes with covariance functions $I \delta(t-\tau)$. The criterion

$$
J=E\left(\lim _{T \rightarrow \infty} \frac{1}{T} \int_{0}^{T}\left(x^{T} C^{T} C x+u^{T} u\right) d t\right)
$$

is required to be minimized and the corresponding optimal compensator is given by

$$
\dot{z}=A z+B u+S C^{T}(y-C z), \quad u=-B^{T} P z
$$

where $S$ is the stabilizing solution (i.e. $\sigma\left(A-S C^{T} C\right) \subset \mathrm{C}^{-}$) to the Filter Algebraic Riccati Equation (FARE)

$$
A S+S A^{T}+B B^{T}-S C^{T} C S=0
$$

and $P$ is the stabilizing solution (i.e. $\sigma\left(A-B B^{T} P\right) \subset \mathrm{C}^{-}$) to the control algebraic Riccati equation (CARE)

$$
A^{T} P+P A+C^{T} C-P B B^{T} P=0
$$


Theorem 2.1 ([6,12]) The eigenvalues of PS are similarity invariants and there exists a state space representation where

$$
M:=P=S=\left(\begin{array}{ccc}
\mu_{1} & & 0 \\
& \ddots & \\
0 & & \mu_{n}
\end{array}\right)
$$

with $\mu_{1} \geq \ldots \geq \mu_{n}>0$. This is called a $L Q G$ balanced representation.

In [6] and [12], it is argued that if $\mu_{k}>>\mu_{k+1}$, then the state components $x_{1}$ up to $x_{k}$ are more difficult both to control and to filter than $x_{k+1}$ up to $x_{n}$ and a synthesis based only on $x_{1}, . ., x_{k}$ probably retains the stability and sensitivity properties of the system. If we assume system (1) is LQG balanced, then the reduced order system is

$$
\dot{x}=A_{11} x+B_{1} u+B_{1} d \quad y=C_{1} x+v
$$

where the entries of $A_{11}$ form the first $k$ columns and rows of $A$, the entries of $B_{1}$ are the first $k$ rows of $B$ and the entries of $C_{1}$ are the first $k$ columns of $C$.

Theorem 2.2 ([6, 12]) If $\left(A_{11}, B_{1}, C_{1}\right)$ is minimal the reduced order system (7) is $L Q G$ balanced again and the optimal compensator for system (7) is the reduced order optimal compensator of the full order system (1).

The original idea of balancing for stable linear systems, introduced in [9], considers the Hankel singular values which are a measure of the importance of a state component. This is based on the input energy which is necessary to reach this state component and the output energy which is generated by this state component. A similar kind of reasoning, using a different pair of energy functions, can be used to achieve the similarity invariants $\mu_{i}, i=1, . ., n$, as above, see [18]. For this we consider a minimal system

$$
\dot{x}=A x+B u, \quad y=C x
$$

where $u \in \mathbf{R}^{m}, x \in \mathbf{R}^{n}$ and $y \in \mathbf{R}^{p}$ (N.B.: no noise is entering the system). We define the following energy functions

$$
\begin{aligned}
& K^{-}\left(x_{0}\right):=\min _{\substack{u \in L_{2}(-\infty, 0) \\
x(-\infty)=0, x(0)=x_{0}}} \frac{1}{2} \int_{-\infty}^{0}\left(\|y(t)\|^{2}+\|u(t)\|^{2}\right) d t \\
& K^{+}\left(x_{0}\right):=\min _{\substack{u \in L_{2}(0, \infty) \\
x(\infty)=0, x(0)=x_{0}}} \frac{1}{2} \int_{0}^{\infty}\left(\|y(t)\|^{2}+\|u(t)\|^{2}\right) d t
\end{aligned}
$$

$K^{-}\left(x_{0}\right)$ will be called the past energy and $K^{+}\left(x_{0}\right)$ the future energy of the system in the state $x_{0}$.

Theorem 2.3 ([18]) $K^{-}(x)=\frac{1}{2} x^{T} S^{-1} x$ and $K^{+}(x)=\frac{1}{2} x^{T} P x$, where $S$ and $P$ are the stabilizing solutions of respectively (4) and (5).

For the LQG balanced representation from Theorem 2.1 the past and future energy function are respectively $K^{-}\left(x_{0}\right)=\frac{1}{2} x_{0}^{T} M^{-1} x_{0}$ and $K^{+}\left(x_{0}\right)=\frac{1}{2} x_{0}^{T} M x_{0}$, where $M$ is diagonal. Then the importance of the state $\tilde{x}=\left(0 \ldots 0 x_{i} 0 \ldots 0\right)$ in terms of past and future energy can be measured by the similarity invariant $\mu_{i}$. For large $\mu_{i}$ the influence of the state $\tilde{x}$ on the future energy is large while the influence on the past energy is small. Hence if $\mu_{k}>>\mu_{k+1}$, the state components $x_{k+1}$ to $x_{n}$ are not important from this energy point of view and can be removed to reduce the number of state components of the model.

\subsection{Balancing of the coprime representation}

In [8] and [11] balancing of the coprime representation of a linear system is dealt with. Here we will give a very brief review of this subject. Consider the following system which is a state representation of the so called Graph operator of system (8)

$$
\dot{x}=\left(A-B B^{T} P\right) x+B w,\left(\begin{array}{l}
y \\
u
\end{array}\right)=\left(\begin{array}{c}
C \\
-B^{T} P
\end{array}\right) x+\left(\begin{array}{l}
0 \\
I
\end{array}\right) v
$$

with $w$ a (fictitious) input variable and $P$ is the stabilizing solution to (5). This Graph operator is representing the normalized right coprime factorization of system (8), see [8]. The Hankel singular values of system (9) (for a definition of Hankel singular values see i.e. [2]) are called the Graph Hankel singular values of system (8), and have the following property

Theorem $2.4([8,11])$ The Graph Hankel singular values of system (8) are strictly less than one.

Now denote the Graph Hankel singular values by $\tau_{i}, i=1, . ., n$, and assume $\tau_{1} \geq \ldots \geq \tau_{n}$. The relation between $\tau_{i}, i=1, \ldots, n$, and the similarity invariants $\mu_{i}, i=1, . ., n$, of Theorem 2.1 is given by the following theorem:

Theorem $2.5([11,18]) \mu_{i}=\tau_{i}\left(1-\tau_{i}^{2}\right)^{-\frac{1}{2}}$ for $i=1, . ., n$.

In particular, this means that the reduced model that is obtained by model reduction based on the concept of balancing the normalized right coprime factorization will be the same as the reduced model that is obtained by model reduction based on the concept of LQG balancing.

\section{Review for nonlinear systems}

\subsection{Balancing for stable nonlinear systems}

Balancing for stable nonlinear systems is dealt with in [14]. As in the linear case, this is a method based on the input energy that is necessary to reach a state and the output energy that is generated by this state. We will give a brief review on this subject in this section.

Consider a smooth, i.e., $C^{\infty}$, nonlinear system of the form

$$
\dot{x}=f(x)+g(x) u, \quad y=h(x)
$$

where $u=\left(u_{1}, . ., u_{m}\right) \in \mathbf{R}^{m}, y=\left(y_{1}, . ., y_{p}\right) \in \mathbf{R}^{p}$ and $x=$ $\left(x_{1}, \ldots, x_{n}\right)$ are local coordinates for a smooth state space manifold denoted by $M$. Throughout we assume that the system has an equilibrium. Without loss of generality we take this equilibrium in 0 , i.e. $f(0)=0$ and we also take $h(0)=0$.

Definition 3.1 The controllability and observability function of a nonlinear system are defined as

$$
L_{c}\left(x_{0}\right)=\min _{\substack{u \in L_{2}(-\infty, 0) \\ x(-\infty)=0, x(0)=x_{0}}} \frac{1}{2} \int_{-\infty}^{0}\|u(t)\|^{2} d t
$$

respectively

$$
L_{o}\left(x_{0}\right)=\frac{1}{2} \int_{0}^{\infty}\|y(t)\|^{2} d t, \quad x(0)=x_{0}, \quad u(t) \equiv 0
$$

The value of the controllability function at $x_{0}$ is the minimum amount of control energy required to reach the state $x_{0}$ and the value of the observability function at $x_{0}$ is the amount of output energy generated by $x_{0}$. These functions do not necessarily exist (i.e. are finite), in particular, $L_{o}$ can be infinite if the system is 
unstable and if $x_{0}$ can not be reached from 0 , then by convention $L_{c}\left(x_{0}\right)$ will be infinite. We throughout assume $L_{c}$ and $L_{o}$ are finite on some neighborhood $W$ of 0 . Also, for the rest of this paper we assume $L_{c}$ and $L_{o}$ are smooth functions of $x$.

Theorem 3.2 ([14]) If $f(x)$ is asymptotically stable on a neighborhood $W$ of 0 , then for all $x \in W, L_{o}(x)$ is the unique smooth solution of the following Hamilton-Jacobi equation:

$$
\frac{\partial L_{o}}{\partial x}(x) f(x)+\frac{1}{2} h^{T}(x) h(x)=0, \quad L_{o}(0)=0
$$

Furthermore for all $x \in W, L_{c}(x)$ is the unique smooth solution of the following Hamilton-Jacobi equation:

$$
\frac{\partial L_{c}}{\partial x}(x) f(x)+\frac{1}{2} \frac{\partial L_{c}}{\partial x}(x) g(x) g^{T}(x) \frac{\partial^{T} L_{c}}{\partial x}(x)=0, \quad L_{c}(0)=0
$$

satisfying $-\left(f(x)+g(x) g^{T}(x) \frac{\partial^{T} \bar{L}_{c}}{\partial x}(x)\right)$ is asymptotically stable on $W$

Remark 3.3 ([14]) $L_{c}$ and $L_{o}$ are non-negative.

Theorem 3.4 ([14]) Assume $f$ is asymptotically stable on $W$ and (14) has a smooth solution $\bar{L}_{c}$ on $W$. Then $\bar{L}_{c}\left(x_{0}\right)>0$ for $x_{0} \in W, x_{0} \neq 0$, if and only if $-\left(f(x)+g(x) g^{T}(x) \frac{\partial^{T} L_{c}}{\partial x}(x)\right)$ is asymptotically stable on $W$.

For the following definition see e.g. [13].

Definition 3.5 The system (10) is reachable from $x_{0}$ if for any $\bar{x} \in M$ there exists a $\bar{t} \geq 0$ and input $u$ such that $\bar{x}=\varphi\left(\bar{t}, 0, x_{0}, u\right)$. The system (10) is zero-state observable if any trajectory where $u(t) \equiv 0, y(t) \equiv 0$ implies $x(t) \equiv 0$, i.e., $h(\varphi(t, 0, x, 0))=0$, $t \geq 0 \Rightarrow \varphi(t, 0, x, 0)=0, t \geq 0$, for all $x \in M$.

The following theorem is closely related to some results in [4] and [13]. For the proof, see [14].

Theorem 3.6 Assume $f(x)$ is asymptotically stable on a neigh borhood $W$ of 0 . If the system (10) is zero-state observable and (13) has on $W$ the smooth solution $L_{o}$, then $L_{o}\left(x_{0}\right)>0, \forall x_{0} \in$ $W, x_{0} \neq 0$.

Now we consider nonlinear systems of the form (10) with controllability and observability function $L_{c}$ respectively $L_{o}$ as in definition 3.1, and with the following standing assumptions:

1. $f(x)$ is asymptotically stable on a neighborhood $Y$ of 0

2. the system is zero-state observable on $Y$

3. $L_{g}$ and $L_{c}$ are smooth and finite on $Y$

4. $\frac{\partial^{2} L_{c}}{\partial x^{2}}(0)>0$ and $\frac{\partial^{2} L_{o}}{\partial x^{2}}(0)>0$

To balance the nonlinear system we first need the following lemma:

Lemma 3.7 ([14]) There exists a coordinate transformation $x=$ $\phi(\bar{x}), \phi(0)=0$, such that $L_{c}(x)$ in the new coordinates $\bar{x}=$ $\phi^{-1}(x)$ is of the form $L_{c}(\phi(\bar{x}))=\frac{1}{2} \bar{x}^{T} \bar{x}$. Furthermore we can write $L_{o}(x)$ in the new coordinates $\bar{x}=\phi^{-1}(x)$ as $L_{o}(\phi(\bar{x}))=$ $\frac{1}{2} \bar{x}^{T} M(\bar{x}) \bar{x}$ where $M(0)=\frac{\partial^{2} L_{o}}{\partial x^{2}}(0)$, with $M(\bar{x}) a n \times n$ symmetric matrix with entries which are smooth functions of $\bar{x}$.

Theorem 3.8 ([14]) Consider system (10) and assume there exists a neighborhood $V$ of 0 where the number of distinct eigenval ues of $M(\bar{x})$ is constant for $\bar{x} \in V$. Then there exists a neighborhood $U$ of 0 and a coordinate transformation $x=\psi(z), \psi(0)=0$, such that $L_{c}(x)$ in the new coondinates $z \in W:=\psi^{-1}(U)$ is of the following form:

$$
\tilde{L}_{c}(z):=L_{c}(\psi(z))=\frac{1}{2} z^{T} z
$$

while in the new coordinates $L_{o}$ is of the following form:

$$
\tilde{L}_{o}(z):=L_{\circ}(\psi(z))=\frac{1}{2} z^{T}\left(\begin{array}{ccc}
\tau_{1}(z) & & 0 \\
& \ddots & \\
0 & & \tau_{n}(z)
\end{array}\right) z
$$

where $\tau_{1}(z) \geq \ldots \geq \tau_{n}(z)$ are smooth functions of $z$, called the singular value functions.

Remark 3.9 For a linear system the singular value functions $\tau_{i}, i=1, \ldots, n$ are constant and are equal to the squared Hankel singular values.

The form of the controllability and observability function in (15) and (16) is not yet entirely balanced. For that we need another additional coordinate transformation. We take as transformation $\bar{z}_{i}=\eta_{i}\left(z_{i}\right):=\tau_{i}\left(0, . ., 0, z_{i}, 0, . .0\right)^{\frac{1}{2}} z_{i}, i=1, . ., n$ and hence $\bar{z}=\eta(z):=\left(\eta_{1}\left(z_{1}\right) \quad \ldots \quad \eta_{n}\left(z_{n}\right)\right)$ on $\bar{z} \in \bar{W}:=\eta(W)$. Define $\bar{L}_{c}(\bar{z}):=\tilde{L}_{c}\left(\eta^{-1}(\bar{z})\right)$ and $\bar{L}_{o}(\bar{z}):=\tilde{L}_{o}\left(\eta^{-1}(\bar{z})\right)$. Then $(15)$ and (16) become respectively:

$$
\begin{aligned}
& \bar{L}_{c}(\bar{z})=\frac{1}{2} \bar{z}^{T}\left(\begin{array}{ccc}
\sigma_{1}\left(\bar{z}_{1}\right)^{-1} & & 0 \\
& \ddots & \\
0 & & \sigma_{n}\left(\bar{z}_{n}\right)^{-1}
\end{array}\right) \bar{z} \\
& \bar{L}_{o}(\bar{z})=\frac{1}{2} \bar{z}^{T}\left(\begin{array}{ccc}
\omega_{1}(\bar{z}) & & 0 \\
& \ddots & \\
0 & & \omega_{n}(\bar{z})
\end{array}\right) \bar{z}
\end{aligned}
$$

where for $i=1, . ., n$ we have $\sigma_{i}\left(\bar{z}_{i}\right)=\tau_{i}\left(0, . ., 0, \eta_{i}^{-1}\left(\bar{z}_{i}\right), 0, . ., 0\right)^{\frac{1}{2}}$ and $\omega_{i}(\bar{z})=\sigma_{i}\left(\bar{z}_{i}\right)^{-1} \tau_{i}\left(\eta^{-1}(\bar{z})\right)$. It follows that $\bar{L}_{c}\left(0, \ldots, 0, \bar{z}_{i}, 0, \ldots, 0\right)$ $=\frac{1}{2} \bar{z}_{i}^{2} \sigma_{i}\left(\bar{z}_{i}\right)^{-1}$ and $\bar{L}_{o}\left(0, . ., 0, \bar{z}_{i}, 0, \ldots, 0\right)=\frac{1}{2} \bar{z}_{i}^{2} \sigma_{i}\left(\bar{z}_{i}\right)$ for $i=1, . ., n$. We call a nonlinear system balanced if it has a controllability and observability function of the form of respectively (17) and (18). This means that we can balance system (10) by a coordinate transformation of the form $x=\chi(\bar{z}):=\psi\left(\eta^{-1}(\bar{z})\right)$ where $\psi$ is as in Theorem 3.8.

\subsection{Balancing of the coprime factorization}

If the nonlinear system is not stable, we can consider the normalized right coprime factorization of a nonlinear system, and apply the theory of section 3.1 to the normalized right coprime representation, see [15]. We will give a brief review. Consider a system of the form (10), i.e.

$$
\dot{x}=f(x)+g(x) u, \quad y=h(x)
$$

with the same properties as (10) and let the system be zero-state observable. For the normalized right coprime representation of this system we consider the following Hamilton-Jacobi-Bellman equation (known from optimal control theory):

$$
\frac{\partial V}{\partial x}(x) f(x)-\frac{1}{2} \frac{\partial V}{\partial x}(x) g(x) g(x)^{T} \frac{\partial^{T} V}{\partial x}(x)+\frac{1}{2} h(x)^{T} h(x)=0
$$

with $V(0)=0$. Assume (20) has a smooth proper positive definite solution $V$. Then the following system is a representation of the normalized right coprime factorization of system (19) (see for details (15])

$$
\begin{aligned}
& \dot{x}=\left(f(x)-g(x) g(x)^{\mathrm{T}} \frac{\partial^{T} V}{\partial x}(x)\right)+g(x) w \\
& \left\{\begin{array}{l}
y=h(x) \\
u=-g(x)^{T} \frac{\partial^{T} V}{\partial x}(x)+w
\end{array}\right.
\end{aligned}
$$


In the linear case this is the state space representation of the Graph operator (see section 2.2). The following lemmas are related to results from e.g. [7]:

Lemma 3.10 ([15]) System (21) is zero-state observable.

Lemma 3.11 ([15]) Let $V$ be a smooth positive definite solution to the Hamilton-Jacobi-Bellman equation (20), then $\dot{x}=f(x)-$ $g(x) g(x)^{T} \frac{\partial^{T} V}{\partial x}(x)$ is locally asymptotically stable. If $V$ is proper, then $\dot{x}=f(x)-g(x) g(x)^{T} \frac{\partial^{T} V}{\partial x}(x)$ is globally asymptotically stable.

From [15] we know that the observability function $\bar{L}_{o}$ of system (21) is well defined and is the smooth positive definite solution of $(20)$, hence $\bar{L}_{o}=V$. Additionally let us assume that the controllability function $\bar{L}_{c}$ of system (21) is smooth and finite, then $\bar{L}_{c}$ fulfills

$$
\frac{\partial \bar{L}_{c}}{\partial x}(x)\left(f-g(x) g(x)^{T} \frac{\partial^{T} L_{o}}{\partial x}(x)\right)+\frac{1}{2} \frac{\partial L_{c}}{\partial x}(x) g(x) g(x)^{T} \frac{\partial^{T} L_{c}}{\partial x}=0
$$

satisfying $-\left(f(x)+g(x) g(x)^{T} \frac{\partial^{T}\left(L_{c}-L_{o}\right)}{\partial x}(x)\right)$ is asymptotically stable. To apply the theory of section 3.1 we also assume that $\frac{\partial^{2} L_{o}}{\partial x^{2}}(0)>0$ and $\frac{\partial^{2} L_{c}}{\partial x^{2}}(0)>0$. We can apply Theorem 3.8 to the system (21) and we call the singular value functions of this system the Graph singular value functions of the original system (19). The following result can be compared with Theorem 2.4 for linear systems.

Theorem 3.12 ([15]) $\bar{L}_{o} \leq \bar{L}_{c}$.

In particular, this implies that the Graph singular value functions $\tilde{\tau}_{i}(x), i=1, . ., n$, of a nonlinear system satisfy $\tilde{\tau}_{i}\left(0, . ., 0, x_{i}, 0, . ., 0\right)$ $\leq 1$ for $i=1, \ldots, n$.

\section{Balancing for unstable nonlinear systems}

\subsection{The HJB balanced form}

For closed loop balancing we first follow the idea of [18] which has been treated briefly in section 2.1. Consider the system (10)

$$
\dot{x}=f(x)+g(x) u, \quad y=h(x)
$$

We assume the system is zero-state observable. First we define the following energy functions (see also section 2.1):

Definition 4.1 The past and future energy function of a nonlinear system are defined as

$$
K^{-}\left(x_{0}\right)=\min _{\substack{u \in L_{2}(-\infty, 0) \\ x(-\infty)=0, x(0)=x_{0}}} \frac{1}{2} \int_{-\infty}^{0}\left(\|y(t)\|^{2}+\|u(t)\|^{2}\right) d t
$$

respectively

$$
K^{+}\left(x_{0}\right)=\min _{\substack{u \in L_{2}(0, \infty) \\ x(\infty)=0, x(0)=x_{0}}} \frac{1}{2} \int_{0}^{\infty}\left(\|y(t)\|^{2}+\|u(t)\|^{2}\right) d t
$$

We assume that $K^{-}\left(x_{0}\right)$ and $K^{+}\left(x_{0}\right)$ are smooth and finite. From optimal control theory we know that $K^{+}$is the smooth nonnegative solution to the Hamilton-Jacobi-Bellman equation (20) from section 3.2. $K^{+}$is minimized by $u=-g(x)^{T} \frac{\partial^{T} K^{+}}{\partial x}(x)$.

Theorem 4.2 $\mathrm{K}^{+}$is the smooth non-negative solution to the following Hamilton-Jacobi-Bellman equation:

$$
\frac{\partial K^{+}}{\partial x}(x) f(x)-\frac{1}{2} \frac{\partial K^{+}}{\partial x}(x) g(x) g(x)^{T} \frac{\partial^{T} K^{+}}{\partial x}(x)+\frac{1}{2} h^{T}(x) h(x)=0
$$

with $K^{+}(0)=0$ and satisfying $f(x)-g(x) g(x)^{T} \frac{\partial^{T} K^{+}}{\partial x}(x)$ is asymptotically stable. Furthermore, $K^{+}$is the smooth non-negative solution to the following Hamilton-Jacobi-Bellman equation:

$\frac{\partial K^{-}}{\partial x}(x) f(x)+\frac{1}{2} \frac{\partial K^{-}}{\partial x}(x) g(x) g^{T}(x) \frac{\partial^{T} K^{-}}{\partial x}(x)-\frac{1}{2} h(x)^{T} h(x)=0(27)$ with $K^{-}(0)=0$ and satisfying $-\left(f(x)+g(x) g(x)^{T} \frac{\partial^{T} K^{-}}{\partial x}(x)\right)$ is asymptotically stable.

Remark 4.3 The existence of a smooth solution of (26) satisfying the condition on asymptotic stability, is equivalent with the existence of $K^{+}$. The same is valid for (27) and $K^{-}$, see cf. [7].

Theorem 4.4 Assume (26) has a smooth proper solution $K$ on $W$. Then $K\left(x_{0}\right)>0$ for $x_{0} \in W, x_{0} \neq 0$, if and only if $f(x)-g(x) g(x)^{r} \frac{\partial^{T} K}{\partial x}(x)$ is asymptotically stable on $W$. Similarly, assume (27) has a smooth proper solution $\bar{K}$ on $W$, then $\bar{K}\left(x_{0}\right)>$ 0 for $x_{0} \in W, x_{0} \neq 0$, if and only if $-\left(f(x)+g(x) g(x)^{T} \frac{\partial^{T} R}{\partial x}(x)\right)$ is asymptotically stable on $W$.

Proof Assume $K>0$, then $f(x)-g(x) g(x)^{T} \frac{\partial^{T} K}{\partial x}(x)$ is asymptotically stable on $W$ by Lemma 3.11 .

Now assume $f(x)-g(x) g(x)^{T} \frac{\partial^{T} K}{\partial x}(x)$ is asymptotically stable on $W$. By Theorem 4.2 we know that $K=K^{+}$on $W$, where $K^{+}$is the future energy function of system (23).

$$
K^{+}\left(x_{0}\right)=\min _{\substack{u \in L_{2}(0, \infty) \\ x(\infty)=0, x(0)=x_{0}}} \frac{1}{2} \int_{0}^{\infty}\left(\|y(t)\|^{2}+\|u(t)\|^{2}\right) d t
$$

Furthermore we know from optimal control theory that the minimum is taken for $u=-g(x)^{T} \frac{\partial^{T} K^{+}}{\partial x}(x)$. Hence

$$
K^{+}\left(x_{0}\right)=\frac{1}{2} \int_{0}^{\infty} \frac{\partial K^{+}}{\partial x}(x) g(x) g(x)^{T} \frac{\partial^{T} K^{+}}{\partial x}(x)+h(x)^{T} h(x) d t
$$

Now let $x_{0} \neq 0$. If $\frac{\partial K^{+}}{\partial x}(x) g(x) g(x)^{T} \frac{\partial^{T} K^{+}}{\partial x}(x)+h(x)^{T} h(x)=0$ for $0 \leq t<\infty$ then $u(t)=0$ and $h(x(t))=0$, for all $t, 0 \leq t<\infty$. But by the zero-state observability of system (23) this means that $x(t)=0$ for all $0 \leq t<\infty$ and this contradicts $x_{0} \neq 0$. Hence $K^{+}\left(x_{0}\right)>0, \forall x_{0} \in W, x_{0} \neq 0$.

The second part of the theorem can be proven by using the same type of arguments.

Now we consider nonlinear systems of the form (23) with future and past energy function respectively $K^{+}$and $K^{-}$smooth functions as in definition 4.1, with the following assumptions:

1. $K^{+}$and $K^{-}$are finite on some neighborhood $Y$ of 0

2. $\frac{\partial^{2} K^{+}}{\partial x^{2}}(0)>0$ and $\frac{\partial^{2} K^{-}}{\partial x^{2}}(0)>0$

3 . the system is zero-state observable on $Y$

Similar to Lemma 3.7 and Theorem 3.8 we can bring $\mathrm{K}^{+}$and $\mathrm{K}^{-}$ into a special form:

Lemma 4.5 There exists a coordinate transformation $x=\varphi(\bar{x})$, $\varphi(0)=0$, such that $K^{-}(x)$ in the new coordinates $\bar{x}=\varphi^{-1}(x)$ is of the form $K^{-}(\varphi(\bar{x}))=\frac{1}{2} \bar{x}^{T} \bar{x}$. Furthermore we can write $K^{+}(x)$ in the new coordinates $\bar{x}=\varphi^{-1}(x)$ in the form $K^{+}(\varphi(\bar{x}))=$ $\frac{1}{2} \bar{x}^{T} N(\bar{x}) \bar{x}$ with $N(0)=\frac{\partial^{2} K^{+}}{\partial x^{2}}(0)$, where $N(\bar{x})$ is a $n \times n$ symmetric matrix with entries which are smooth functions of $\bar{x}$.

Proof This follows the proof of Lemma 3.7.

Theorem 4.6 Consider system (23) and assume there exists a neighborhood $V$ of 0 where the number of distinct eigenvalues of $N(\bar{x})$ is constant for $\bar{x} \in V$. On a neighborhood $U$ of zero there exists a coordinate transformation $x=\gamma(z), \gamma(0)=0$, such that $K^{-}(x)$ in the new coordinates $z \in W:=\gamma^{-1}(U)$ is of the following form: 


$$
\tilde{K}^{-}(z):=K^{-}(\gamma(z))=\frac{1}{2} z^{T} z
$$

while in the new coondinates $K^{+}$is of the following form:

$$
\tilde{K}^{+}(z):=K^{+}(\gamma(z))=\frac{1}{2} z^{T}\left(\begin{array}{ccc}
v_{1}(z) & & 0 \\
& \ddots & \\
0 & & v_{n}(z)
\end{array}\right) z
$$

where $v_{1}(z) \geq \ldots \geq v_{n}(z)$ are smooth functions of $z$, called the HJB singular value functions (HJB stands for Hamilton-JacobiBellman).

Proof This follows the proof of Theorem 3.8.

Remark 4.7 For linear systems the HJB singular value functions $v_{i}, i=1, . ., n$ are constant and are equal to the squared similarity invariants of Theorem 2.1 .

Like in section 3.1 the form of the past and future energy function in (28) and (29) is not yet entirely the form we want. For that we need an additional coordinate transformation. We take as smooth transformation $\bar{z}_{i}=\xi_{i}\left(z_{i}\right):=v_{i}\left(0, \ldots, 0, z_{i}, 0, .0\right)^{\frac{1}{t}} z_{i}, i=1, \ldots, n$ and hence $\bar{z}=\xi(z):=\left(\begin{array}{lll}\xi_{1}\left(z_{1}\right) & \ldots & \xi_{n}\left(z_{n}\right)\end{array}\right)$ on $\bar{z} \in \bar{W}:=\xi(W)$. Define $\bar{K}^{-}(\bar{z}):=\tilde{K}^{-}\left(\xi^{-1}(\bar{z})\right)$ and $\bar{K}^{+}(\bar{z}):=\tilde{K}^{+}\left(\xi^{-1}(\bar{z})\right)$. Then $(28)$ and (29) become respectively:

$$
\begin{aligned}
& \bar{K}^{-}(\bar{z})=\frac{1}{2} \bar{z}^{T}\left(\begin{array}{ccc}
\mu_{1}\left(\bar{z}_{1}\right)^{-1} & & 0 \\
& \ddots & \\
0 & & \mu_{n}\left(\bar{z}_{n}\right)^{-1}
\end{array}\right) \bar{z} \\
& \bar{K}^{+}(\bar{z})=\frac{1}{2} \bar{z}^{T}\left(\begin{array}{ccc}
\tilde{\omega}_{1}(\bar{z}) & & 0 \\
& \ddots & \\
0 & & \tilde{\omega}_{n}(\bar{z})
\end{array}\right) \bar{z}
\end{aligned}
$$

where for $i=1, . ., n, \mu_{i}\left(\bar{z}_{i}\right)=v_{i}\left(0, . ., 0, \xi_{i}^{-1}\left(\bar{z}_{i}\right), 0, . ., 0\right)^{\frac{1}{2}}$ and $\tilde{\omega}_{i}=\mu_{i}\left(\bar{z}_{i}\right)^{-1} v_{i}\left(\xi^{-1}(\bar{z})\right)$. It follows that $\bar{K}^{-}\left(0, \ldots, 0, \bar{z}_{i}, 0, \ldots, 0\right)=$ $\frac{1}{2} \bar{z}_{i}^{2} \mu_{i}\left(\bar{z}_{i}\right)^{-1}$ and $\bar{K}^{+}\left(0, \ldots, 0, \bar{z}_{i}, 0, \ldots, 0\right)=\frac{1}{2} \bar{z}_{i}^{2} \mu_{i}\left(\bar{z}_{i}\right)$ for $i=1, \ldots, n$. In terms of the past and future energy we can infer from $v_{i}\left(\xi^{-1}(\bar{z})\right)$ $>v_{i+1}\left(\xi^{-1}(\bar{z})\right)$ that the state component $\bar{z}_{i}$ is more important than the state component $\bar{z}_{i+1}$ on $\bar{W}$. We call a nonlinear system $H J B$ balanced if it has a past and future energy function respectively of the form (30) and (31). This means that we can bring system (23) in a HJB balanced form by a coordinate transformation of the form $x=\alpha(\bar{z}):=\gamma\left(\xi^{-1}(\bar{z})\right)$ where $\gamma$ is as in Theorem 4.6. For a linear system this means that the system is in the LQG balanced form, since then $\bar{K}^{-}(\bar{z})=\frac{1}{2} \bar{z}^{T} S^{-1} \bar{z}$ and $\bar{K}^{+}(\bar{z})=\frac{1}{2} \bar{z}^{T} P \bar{z}$ with $P=S=M$ as in Theorem 2.1 .

For linear systems HJB balancing is the same as LQG balancing. However, the formulation of LQG balancing for linear systems can not be extended easily to nonlinear systems. This is nevertheless an interesting problem to consider. The usual stochastic formulation of the LQG problem seems not to be the right formulation of this problem for nonlinear systems. However, there exists a deterministic formulation of the LQG problem, which is equivalent to the stochastic formulation, and which has been extended to nonlinear systems, see Hijab [3]. Consider the following system:

$$
\dot{x}=f(x)+g(x) u, \quad x(0)=x_{0}, \quad y=h(x)+v
$$

where $v$ is the noise that enters the system. We want to minimize the following energy functional:

$$
J\left(x_{0}, u(t), v(t)\right)=W_{0}\left(x_{0}\right)+\frac{1}{2} \int_{0}^{t}\|u(\tau)\|^{2}+\|v(\tau)\|^{2} d \tau
$$

over the input triple $\left(x_{0}, u(\cdot), v(\cdot)\right)$, which corresponds uniquely with an input pair $\left(x_{0}, u(\cdot)\right)$, when the observations $y(\cdot)$ are given, see [3]. $W_{0}\left(x_{0}\right)$ is a real-valued function representing the initial costs, with $W_{0}(0)=0$. Let $\hat{x}(t) \in \mathbf{R}^{n}$ be a deterministic estimate of the state at time $t, t \geq 0$, given the observations $y(\tau), 0 \leq \tau \leq$ $t$ (i.e. $\hat{x}(t)$ is the endpoint of the state trajectory of an input pair $\left(\hat{x}_{0}, \hat{u}(\cdot)\right)$ that is minimizing the energy functional (33) based on the observations). Let $W_{0}$ generate a non-degenerate estimate $\hat{x}(0)$, i.e. $\operatorname{det}\left(\frac{\partial^{2} W_{0}}{\partial x^{2}}(\hat{x}(0))\right) \neq 0$, then for small $t \geq 0$ and for $x$ near $\hat{x}(0)$ we will have $\operatorname{det}\left(\frac{\partial^{2} W}{\partial x^{2}}(t, x)\right) \neq 0$. Now the dynamics of the deterministic estimate $\hat{x}(t)$ is given by (see [3]):

$$
\dot{\hat{x}}=f(\hat{x})+g(\hat{x}) u+\left(\frac{\partial^{2} W}{\partial x^{2}}(t, \hat{x})\right)^{-1}\left(\frac{\partial h}{\partial x}(\hat{x})\right)^{T}(y(t)-h(\hat{x}))
$$

where $W(t, x)$ is a smooth solution of the Mortensen equation:

$$
\begin{aligned}
& \frac{\partial W}{\partial t}+h(x) y(t)+\frac{\partial W}{\partial x} f(x)+\frac{1}{2} \frac{\partial W}{\partial x} g(x) g(x)^{T} \frac{\partial^{T} W}{\partial x} \\
& -\frac{1}{2} h(x)^{T} h(x)=0,
\end{aligned}
$$

$W(0, \cdot)=W_{0}(\cdot)$, and $V$ is the smooth positive solution of the Hamilton-Jacobi-Bellman equation (26):

$$
\frac{\partial V}{\partial x}(x) f(x)-\frac{1}{2} \frac{\partial V}{\partial x}(x) g(x) g(x)^{T} \frac{\partial^{T} V}{\partial x}(x)+\frac{1}{2} h^{T}(x) h(x)=0
$$

$V(0)=0$. Based on separation principles, this motivates to consider the nonlinear compensator $u=-g(\hat{x})^{T} \frac{\partial^{T} V}{\partial x}(\hat{x})$ together with (34). If system (32) is linear, then (34) is equal to the compensator (3) of section 2.1. In that case $\left(\frac{\partial^{2} W}{\partial x^{2}}(t, x)\right)^{-1}$ is constant, i.e. is equal to the matrix $S$ that is the stabilizing solution of equation (4) (the FARE). This results directly from (35), see [3]. For general nonlinear systems equation (35) does not have a solution such that $\frac{\partial^{2} W}{\partial x^{2}}(t, x)$ is independent of the time $t$. Nevertheless we observe that equation (27) is part of the equation (35). It follows that for $y(t) \equiv 0$ for all $-\infty<t \leq 0, W(t, x)=K^{-}(x)$ is a smooth solution of (35). In this case we can obtain the HJB singular value functions from the solutions $W$ and $V$ of the equations (35) and (36) as is done above for respectively $K^{+}$and $K^{-}$, and then the HJB singular value functions are a measure for the difficulties both to control and filter the corresponding state component.

Like in the linear case, we can use HJB-balancing for model reduction. The HJB singular value functions are a measure for the importance of a state component in terms of the past and future energy functions and, as we argued above, in a less strong sense they are a measure for the difficulties both to control and filter a state component.

\subsection{The relation with balancing of the co- prime representation}

In section 2.2 we discussed balancing of the coprime representation of a linear system. We also gave the relation between the Graph Hankel singular values, $\tau_{i}, i=1, . ., n$, and the similarity invariants $\mu_{i}, i=1, \ldots, n$, which gives the relation between LQG-balancing and balancing of the normalized right coprime representation. For nonlinear systems we will find a similar relation.

Consider a system of the form (23) and assume the assumptions 1 to 3 from section 4.1 are fulfilled. Now we consider the HamiltonJacobi-Bellman equations (26) and (27), which have as smooth solutions the future and past energy function $K^{+}$and $K^{-}$. Furthermore consider the equations (20) and (22) which have as solution the observability respectively controllability function, $\tilde{L}_{\text {o }}$ 
and $\bar{L}_{c}$ of system (21), which is representing the normalized right coprime factorization of the original system (23).

Theorem 4.8 The solutions of (20) and (22) are related to the solutions of (26) and (27) by $K^{+}=\bar{L}_{o}$ and $K^{-}=\bar{L}_{c}-\bar{L}_{o}$.

Proof Obviously (20) and (26) are the same equations and hence $K^{+}=\bar{L}_{o}$. From (22) we obtain:

$$
\begin{aligned}
& \frac{\partial \bar{L}_{c}}{\partial x}(x) f(x)+\frac{1}{2} \frac{\partial\left(\bar{L}_{o}-\bar{L}_{c}\right)}{\partial x}(x) g(x) g(x)^{T} \frac{\partial^{T}\left(L_{o}-\bar{L}_{c}\right)}{\partial x}(x) \\
& -\frac{1}{2} \frac{\partial L_{a}}{\partial x}(x) g(x) g(x)^{T} \frac{\partial^{T} \bar{L}_{o}}{\partial x}(x)=0
\end{aligned}
$$

If we subtract (20) from this, we obtain:

$$
\begin{aligned}
& \frac{\partial\left(L_{c}-L_{o}\right)}{\partial x}(x) f(x)+\frac{1}{2} \frac{\partial\left(L_{c}-L_{o}\right)}{\partial x}(x) g(x) g(x)^{T} \frac{\partial^{T}\left(L_{c}-L_{o}\right)}{\partial x}(x) \\
& -\frac{1}{2} h(x)^{T} h(x)=0
\end{aligned}
$$

and hence $K^{-}=\bar{L}_{c}-\bar{L}_{0}$.

Corollary $4.9 \bar{L}_{o}(x)<\bar{L}_{c}(x)$ for $x \neq 0$.

To find the relation between the HJB singular value functions and the Graph singular value functions, we assume that the representation of the normalized right coprime factorization (21) of system (23) has the form such that the observability and controllability function for $x \in U$ are of the following form:

$$
\bar{L}_{c}(x)=\frac{1}{2} x^{T} x, \quad \bar{L}_{o}(x)=\frac{1}{2} x^{T}\left(\begin{array}{ccc}
\tilde{\tau}_{1}(x) & & 0 \\
& \ddots & \\
0 & & \tilde{\tau}_{n}(x)
\end{array}\right) x
$$

where the $\tilde{\tau}_{i}(x)$ 's are the Graph singular value functions as defined in section 3.2. From Corollary 4.9 we obtain that for $i=$ $1, . ., n, \tilde{\tau}_{i}\left(0, . ., 0, x_{i}, 0, \ldots, 0\right)<1$. Furthermore we assume that for $x \in U$, with $U$ a neighborhood of 0 , the transformation which is necessary to bring the system in the form of Theorem 4.6, is $z=$ $\gamma(x), \gamma(0)=0$, for $z \in W:=\gamma(U)$. Hence $\tilde{K}^{-}(z):=K^{-}\left(\gamma^{-1}(z)\right)$ is of the form (28) and $\tilde{K}^{-}(z):=K^{+}\left(\gamma^{-1}(z)\right)$ is of the form (29) where the $v_{i}(z)$ 's are the HJB singular value functions.

Theorem 4.10 There exists a neighborhood $U$ of 0 such that by the coordinate transformation $z=\gamma(x)$ for all $z \in W=\gamma(U)$ :

$$
v_{i}(z)=\frac{\tilde{\tau}_{i}\left(\gamma^{-1}(z)\right)}{1-\tilde{\tau}_{i}\left(\gamma^{-1}(z)\right)}, \quad i=1, . ., n
$$

Proof Since $\tilde{\tau}_{i}\left(0, . ., 0, x_{i}, 0, . ., 0\right)<1, i=1, . ., n$, and by continuity, there exists a neighborhood $U$ of 0 such that $\tilde{\tau}_{i}(x)<1$ for all $x \in U$. By the forms of the observability and controllability functions we infer that $K^{+}$and $K^{-}$are of the following form:

$$
\begin{aligned}
& K^{+}(x)=\frac{1}{2} x^{T}\left(\begin{array}{ccc}
\tilde{\tau}_{1}(x) & & 0 \\
& \ddots & \\
0 & & \tilde{\tau}_{n}(x)
\end{array}\right) x \\
& K^{-}(x)=\frac{1}{2} x^{T}\left(\begin{array}{ccc}
1-\tilde{\tau}_{1}(x) & & 0 \\
& \ddots & 1-\tilde{\tau}_{n}(x)
\end{array}\right) x
\end{aligned}
$$

It follows that the coordinate transformation necessary to bring $K^{-}$and $K^{+}$in the form of respectively (28) and (29) is given by $z_{i}=\gamma_{i}(x):=\left(1-\tilde{\tau}_{i}(x)\right)^{\frac{1}{2}} x_{i}, i=1, . ., n$ and $z=\gamma(x)=$ $\left(\gamma_{1}(x) \cdots \gamma_{n}(x)\right)$ for $z \in \gamma(U)=W$. This proves the theorem.

From this theorem it is clear that $\tilde{\tau}_{1}\left(\gamma^{-1}(z)\right) \geq \ldots \geq \tilde{\tau}_{n}\left(\gamma^{-1}(z)\right)$ is equivalent with $v_{1}(z) \geq \ldots \geq v_{n}(z)$. Furthermore the form of the transformation $z=\gamma(x)$ in the proof of Theorem 4.10 is such that $x_{k}=0$ is equivalent with $z_{k}=0$ and hence if we want to reduce the order of the original model based on these singular value functions, HJB-balancing and balancing of the coprime representation for $x \in U$ both result in the same reduced order model.

\section{Conclusion}

We introduced a procedure to balance unstable nonlinear systems. The method, called HJB balancing, is an extension of a method to balance unstable linear systems, since we considered the past and the future energy of the system. The procedure based on the past and future energy functions is in the linear case equivalent to LQG balancing, while for nonlinear systems in general this does not hold. Furthermore, we gave a relation between HJB balancing and balancing of the normalized right coprime representation of an unstable nonlinear system.

\section{Acknowledgement}

I would like to thank Arjan van der Schaft for the useful discussions and his advice on this paper.

\section{References}

[1] P.E. Crouch, A.J. van der Schaft, Variational and Hamiltonian control systems, Lect. Notes Contr. Inf. Sci. No. 101, (Springer Verlag, Berlin, 1987).

[2] K. Glover, All optimal Hankel-norm approximations of linear multivariable systems and their $L^{\infty}$-error bounds, Int. J. of Contr. 39 (1984) 1115-1193.

[3] O.B. Hijab, Minimum energy estimation, Doctoral dissertation, University of California, Berkeley (1980)

[4] D. Hill, P. Moylan, The stability of nonlinear dissipative systems, IEEE Trans. Aut. Contr., AC-21 (1976) 708-711.

[5] E.A. Jonckheere, L.M. Silverman, Singular value analysis of deformable systems, J. of Circ., Syst. and Sign. Proc. 1 (1982) 447-470.

[6] E.A. Jonckheere, L.M. Silverman, A new set of invariants for linear systems - Applications to reduced order compensator design, IEEE Trans. Aut. Contr. AC-28 (1983) 953-964.

[7] E.B. Lee, L. Markus, Foundations of optimal control theory, (John Wiley \& Sons, New York, 1967).

[8] D.G. Meyer, A fractional approach to model reduction, Proceedings American Control Conference, Atlanta (1988) 1041-1047.

[9] B.C. Moore, Principle component analysis in linear systems: Controllability, observability and model reduction, IEEE Trans. Aut. Contr., AC-26, (1981) 17-32.

[10] H. Nijmeijer, A.J. van der Schaft, Nonlinear Dynamical Control Systems (Springer-Verlag, New York, 1990).

[11] R. Ober, D. McFarlane, Balanced canonical forms for minimal systems: A normalized coprime factor approach, Linear Algebra and its Applications, (1989) 23-64.

[12] Ph. Opdenacker, E.A. Jonckheere, LQG balancing and reduced LQG compensation of symmetric passive systems, Int. J. Contr., 41 (1985) 73-109.

[13] A.J. van der Schaft, $L_{2}$-gain analysis of nonlinear systems and nonlinear state feedback $\mathcal{H}_{\infty}$ control, IEEE Trans. Aut. Contr., AC-37 (1992) 770-784.

[14] J.M.A. Scherpen, Balancing for nonlinear systems, Syst. \& Contr. Letters 21 (1993) 143-153.

[15] J.M.A. Scherpen, A.J. van der Schaft, Balancing of the normalized right coprime factorization of a nonlinear system, (Mem. No. 1096, Faculty of Applied Math., University of Twente, 1992).

[16] S. Weiland, Balanced representations and approximation of linear systems, Proc. 28th IEEE CDC, (1989) 1334-1336.

[17] S. Weiland, Balancing general quadratic measures on external signals, in Progress in Systems and Control Theory; Realization and Modelling in System Theory; Proc. MTNS-89, Vol 1 (1990) 133-140.

[18] S. Weiland, Theory of approximation and disturbance attenuation for linear systems, Doctoral diss., Rijksuniversiteit Groningen (1991). 